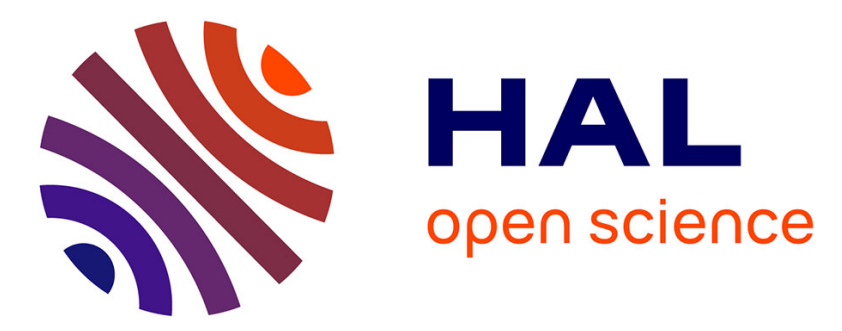

\title{
Avancées thérapeutiques récentes dans la prise en charge du carcinome à cellules de Merkel
}

Pauline Tétu, Barouyr Baroudjian, Isabelle Madelaine, Julie Delyon, Céleste Lebbé

\section{- To cite this version:}

Pauline Tétu, Barouyr Baroudjian, Isabelle Madelaine, Julie Delyon, Céleste Lebbé. Avancées thérapeutiques récentes dans la prise en charge du carcinome à cellules de Merkel. Bulletin du Cancer, 2019, 106, pp.64 - 72. 10.1016/j.bulcan.2018.11.009 . hal-03486685

\section{HAL Id: hal-03486685 \\ https://hal.science/hal-03486685}

Submitted on 20 Dec 2021

HAL is a multi-disciplinary open access archive for the deposit and dissemination of scientific research documents, whether they are published or not. The documents may come from teaching and research institutions in France or abroad, or from public or private research centers.
L'archive ouverte pluridisciplinaire HAL, est destinée au dépôt et à la diffusion de documents scientifiques de niveau recherche, publiés ou non, émanant des établissements d'enseignement et de recherche français ou étrangers, des laboratoires publics ou privés.

\section{()ㅜ(1)}

Distributed under a Creative Commons Attribution - NonCommercial| 4.0 International 


\section{Avancées thérapeutiques récentes dans la prise en charge du carcinome à cellules de Merkel}

\section{Up-date in treatment for Merkel Cell Carcinoma and clinical practice guide}

Pauline Tétu $^{1 *}$, Barouyr Baroudjian ${ }^{1}$, Isabelle Madelaine ${ }^{2}$, Julie Delyon ${ }^{1}$, Céleste Lebbé ${ }^{1}$

${ }^{1}$ APHP Dermatology, Department of Dermatology, Paris 7 Diderot University, INSERM U976, Hôpital Saint-Louis, 1 avenue Claude Vellefaux, 75010 PARIS, France.

${ }^{2}$ APHP Pharmacology, Department of Pharmacology, Paris 7 Diderot University, Hôpital Saint-Louis, 1 avenue Claude Vellefaux, 75010 PARIS, France.

\section{* Corresponding author:}

Pauline TETU ; Dermatology ; Saint-Louis Hospital, 1 avenue Claude Vellefaux, 75010 PARIS ;

mail : pauline.tetu@ hotmail.fr

Funding/support: none

\section{Financial disclosure / Liens d'intérêt:}

Dr. Tétu has nothing to disclose.

Dr Baroudjian has nothing to disclose.

Dr Madeleine has nothing to disclose.

Dr Delyon has nothing to disclose.

Pr. Lebbé reports grants and personal fees from Roche, grants and personal fees from BMS, personal fees from Novartis, personal fees from MSD, personal fees from Amgen, personal fees from Pierre Fabre, personal fees from Pfizer, personal fees from Incyte, outside the submitted work. 


\title{
RÉSUMÉ
}

Le carcinome à cellules de Merkel (MCC) est un cancer neuro-endocrine cutané primitif, rare et agressif associé à des récurrences fréquentes et une mortalité élevée. L'incidence du carcinome à cellules de Merkel a augmenté au cours des dernières années aux États-Unis, en Europe en Australie. Environ 1/3 des patients se présentent avec des métastases au diagnostic ou en développeront au cours de l'évolution de la maladie. Bien que le carcinome à cellules de Merkel avancé soit chimio-sensible, les réponses à la chimiothérapie sont habituellement de courte durée et la toxicité est potentiellement élevée. Récemment, le pronostic du carcinome à cellules de Merkel avancé a été bouleversé par l'arrivée des anti-PD-1 et antiPD-L-1 qui ont démontré une efficacité majeure et durable ainsi qu'un un excellent profil de tolérance. Malgré ces avancées majeures, $50 \%$ des patients au stade avancé ne répondent pas à l'immunothérapie et il existe un besoin urgent d'identifier des facteurs prédictifs de réponse. De plus, de nombreuses pistes thérapeutiques sont actuellement explorées dans le cadre d'études cliniques et l'inclusion dans ces protocoles est encouragée pour les patients au stade métastatique. Cet article synthétise les recommandations actuelles, les avancées thérapeutiques récentes et les traitements en cours d'exploration afin d'aider les cliniciens dans la prise en charge du carcinome à cellules de Merkel.

Mots-clés : carcinome à cellules de Merkel, traitement, immunothérapie, avancées thérapeutiques.

\begin{abstract}
Merkel Cell Carcinoma (MCC) is a rare neuroendocrine skin cancer that is associated with frequent recurrences and a high mortality rate. In the recent past years, incidence rates of MCC have increased in the USA and Europe. About one third of patients present metastatic disease at the time of diagnosis or will develop metastases in the course of their disease. Although advanced MCC is chemo-sensitive, responses to cytotoxic chemotherapy are mostly of short duration and toxicity is potentially high. Recently, considerable progress has been made in the MCC field with the arrival of immunotherapy, particularly anti-PD-1 and antiPD-L-1 antibodies which have demonstrated impressive frequency and durability of response and were well-tolerated. However, about $50 \%$ of advanced patients do not respond to immunotherapy and urgent need exists to identify biomarkers and predictive factors. Moreover, many randomized prospective studies are evaluating the efficacy and safety of novel therapeutics and patients with advanced stages are encouraged to participate in clinical trials. This article synthetizes the actual clinical practice guidelines, the safety and efficacy data from the recent clinical trials and the on-going clinical trials to help clinicians in the treatment of MCC patients.
\end{abstract}

Keywords: merkel cell carcinoma, treatment, immunotherapy, advances.

\section{Introduction}

Le carcinome à cellules de Merkel (MCC) est une tumeur neuro-endocrine cutanée primitive rare et agressive associée à un taux de survie à 10 ans d'environ $50 \%$ en Europe [1]. L'incidence du carcinome à cellules de Merkel augmente rapidement dans le monde [2-5] avec une incidence annuelle estimée actuellement à 2500 cas par an aux USA et devant atteindre 3250 cas par an en 2025 [4]. 
Deux étiologies principales impliquées dans le développement du carcinome à cellules de Merkel ont été décrites : l'intégration du polyomavirus de Merkel (MCPyV) et/ou l'exposition chronique aux UV. Environ $80 \%$ des cas de carcinome à cellules de Merkel en Europe et aux États-Unis sont liés à l'intégration du MCPyV tandis que les cas australiens sont majoritairement liés à l'exposition aux UV [6,7]. Chez les patients MCPyV positifs, les oncoprotéines virales exprimées sont hautement immunogènes pour les lymphocytes $\mathrm{B}$ et $\mathrm{T}$, fournissant un rationnel pour le traitement par immunothérapie. Les tumeurs MCPyV négatives sont liées à l'accumulation de mutations induites par les UV et seraient de plus mauvais pronostic [8]. Les autres facteurs de risque impliqués dans le développement d'un carcinome à cellules de Merkel sont l'âge supérieur à 65 ans et l'immunosuppression (infection VIH, transplantés d'organes et hémopathies malignes) [9].

Le carcinome à cellules de Merkel est une tumeur à haut potentiel métastatique puisqu'au moment du diagnostic, approximativement $30 \%$ des patients présentent un envahissement ganglionnaire et des métastases à distance existent chez environ $10 \%$ des patients [10-12]. Des métastases cérébrales sont décrites chez $10 \%$ des patients au stade IV [13]. Le pronostic du carcinome à cellules de Merkel est hautement associé à l'extension de la maladie au moment du diagnostic [10-12]. La survie des patients de stade métastatique est historiquement faible avec une médiane de survie d'environ 9 mois [11]. Jusqu'en 2017, la chimiothérapie associant classiquement un sel de platine et l'etoposide représentait le traitement de $1^{\text {re }}$ ligne des carcinomes à cellules de Merkel métastatiques avec un taux de réponse globale pouvant atteindre jusqu'à $60 \%$ en $1^{\text {re }}$ ligne $[14,15]$. Cependant, la durée de contrôle de la maladie restait courte avec une médiane de survie globale d'environ 9 mois et une survie sans progression d'environ 3 mois $[14,15]$. De plus, ces traitements présentent une toxicité parfois importante dans cette population cible âgée. L'efficacité des chimiothérapies de $2^{\mathrm{e}}$ ligne ou plus était très faible avec un taux de réponse objective (ORR) de $20 \%$ environ et une PFS de 2 mois seulement [14,15]. Récemment, l'arrivée de l'immunothérapie, en particulier des anti-PDL1 [16-19] et anti-PD-1 [20] a bouleversé le pronostic et la prise en charge du carcinome à cellules de Merkel. Face à ces avancées thérapeutiques récentes, de nouvelles recommandations de prise en charge des patients sont nécessaires afin d'aider les cliniciens à définir la stratégie thérapeutique optimale en particulier pour les carcinomes à cellules de Merkel de stade IV.

L'objectif de cette étude était d'effectuer une synthèse des avancées thérapeutiques récentes dans le carcinome à cellules de Merkel en particulier métastatique et de définir une stratégie thérapeutique basée sur les standards de traitement actuel et l'evidence-based medicine.

Ce travail ne se substitue pas aux recommandations officielles et les cliniciens doivent garder une approche critique et personnalisée face à chaque patient. Nous espérons cependant que ce travail aidera les praticiens à définir un projet thérapeutique personnalisé prenant en compte les avancées thérapeutiques récentes.

\section{Matériels et Méthodes}

Une revue de la littérature à partir de PubMed a été effectuée en utilisant les termes «Merkel Cell Carcinoma ». Tous les articles publiés entre janvier 2016 et juin 2018 en langue anglaise ont été inclus. Les recommandations européennes et américaines ont aussi été étudiées. Le site clinicalTrials.gov a été utilisé afin d'identifier les études cliniques en cours dans le traitement du carcinome à cellules de Merkel.

\section{Résultats}



locorégionale (stade I à III de l'AJCC)

\section{Chirurgie}

La chirurgie est le traitement de première intention des patients présentant un carcinome à cellules de Merkel primitif. Le groupe de travail de l'EADO (European Association of Dermato-Oncology) et de l'EORTC (European Organization of Research and Treatment of Cancer) recommande une résection large du site tumoral, avec des marges de 1 à $2 \mathrm{~cm}$ latéralement et jusqu' au fascia musculaire en profondeur, indépendamment de la taille tumorale [7,9]. L'objectif de ces marges de sécurité est d'assurer l'exérèse des métastases satellites microscopiques [7,9]. En cas d'exérèse incomplète, une reprise chirurgicale est systématiquement recommandée [7,9] puisque le risque de récurrence locale est élevé dans ce cas [11,21]. Pour les cas où la chirurgie est impossible ou lorsqu'elle est refusée par le patient, une radiothérapie locale peut être discutée [7,22,23].

Pour les patients avec atteinte ganglionnaire clinique, une biopsie à l'aiguille fine doit être effectuée afin de confirmer le diagnostic et en cas de positivité, un curage lymphatique doit être systématiquement effectué $[9,22]$.

En l'absence d'atteinte ganglionnaire clinique, le prélèvement du ganglion sentinelle est préconisé quelle que soit la taille initiale de la tumeur [7,9,22] car des métastases ganglionnaires microscopiques sont décrites chez environ $30 \%$ des patients $[7,24,25]$. La présence de métastases ganglionnaire microscopique est un important facteur pronostic ; plusieurs études ont démontré une association significative entre la négativité du ganglion sentinelle et une diminution du risque de récurrence ainsi qu'une augmentation de la survie [26,27]. En cas de présence de micro-métastases, un curage ganglionnaire est habituellement recommandé bien qu'aucune étude prospective n'en ait démontré le bénéfice dans la littérature [9,22]. Dans le cas où la technique du ganglion sentinelle ne pourrait être effectuée, il est recommandé d'effectuer une surveillance rapprochée par échographie ganglionnaire et examen clinique tous les 4 mois [9].

\section{Radiothérapie adjuvante}

Une radiothérapie adjuvante du lit tumoral est systématiquement recommandée en association à la chirurgie locale par les sociétés savantes américaines et européennes afin de diminuer le risque de récidive locale [7,9,27-29].

La radiothérapie adjuvante des aires ganglionnaires de drainage a démontré un bénéfice variable dans la littérature. Dans une étude prospective randomisée, la radiothérapie adjuvante des aires ganglionnaires de drainage chez les patients de stade I était associée à une diminution du risque de récurrence locorégionale sans augmentation de la survie globale [30]. Une étude rétrospective effectuée sur 6908 patients aux États-Unis a montré que la radiothérapie adjuvante en association à la chirurgie permettait une augmentation significative de la survie globale comparée à la chirurgie seule chez les patients de stade I et II ; cependant, ce bénéfice n'était pas retrouvé chez les patients de stade III [29].

Bien que la radiothérapie adjuvante des aires de drainage lymphatique ne puisse être recommandée en systématique face aux bénéfices variable dans la littérature [28-31], elle doit être discutée au sein d'une réunion pluridisciplinaire dans le but d'améliorer le contrôle local de la maladie pour tous les patients quel que soit le statut ganglionnaire et particulièrement pour les patients à haut risque de récidive $[9,22]$.

\section{Chimiothérapie adjuvante}


La chimiothérapie adjuvante n'a pas démontré de bénéfice sur la survie globale [29] et est potentiellement responsable d'une toxicité importante ; elle n'est donc pas recommandée

\section{Immunothérapie adjuvante}

Le bénéfice de l'immunothérapie adjuvante chez les patients à haut risque de récidive est en cours d'évaluation et les résultats sont très attendus. Une étude de phase III contrôlée et randomisée évaluant l'efficacité de l'avelumab en adjuvant chez les patients présentant un carcinome à cellules de Merkel avec atteinte ganglionnaire et traités par chirurgie +/radiothérapie adjuvante est ouverte (NCT03271372). Une autre étude est en cours afin de tester l'immunothérapie adjuvante par nivolumab ou ipilimumab versus observation simple chez les patients avec carcinome à cellules de Merkel de résection complète (NCT02196961).

\section{Prise en charge du carcinome de Merkel métastatique}

\section{Blocage de l'axe anti-PD-1/anti-PD-L-1}

La Table 1 résume les résultats des principaux essais thérapeutiques évaluant les anti-PD-1 et anti-PD-L-1 dans le traitement du carcinome à cellules de Merkel métastatique.

\section{Inhibiteurs de $P D-L-1$}

\section{Avelumab}

L'avelumab est un anticorps monoclonal humain IgG1 anti-PD-L-1. La liaison de l'avelumab à PD-L1 bloque l'interaction entre PD-L1 et les récepteurs PD-1 et B7.1, conduisant à une diminution de l'effet immunosuppresseur de PD-L1 sur les lymphocytes T CD8+ cytotoxiques et ainsi à une restauration de la réponse anti-tumorale cellulaire T. L'avelumab peut aussi via l'ADCC (antibody-dependent cell-mediated cytotoxicity) induire in vitro une lyse cellulaire tumorale via les cellules NK (natural killer) bien que l'implication de cette fonction dans la réponse au traitement n'est pas été démontrée in vivo [32].

Dans une étude de phase 2, 88 patients présentant un carcinome à cellules de Merkel de stade IV réfractaire à la chimiothérapie ont été traités par avelumab $10 \mathrm{mg} / \mathrm{kg}$ toutes les 2 semaines en IV (intra-veineuse) [17,18]. Avec une médiane de suivi de 16.4 mois, l'ORR était de $33 \%$ (réponse complète (RC) : $9 \%$ et réponse partielle (RP) : $23 \%$ ). Il est intéressant de noter que ces réponses étaient rapides (médiane : 6.1 semaines, extrêmes : 6-36), indépendantes du statut PD-L-1 ou MCPyV de la tumeur et durables avec $74 \%$ de réponse persistante à 1 an. La survie sans progression (PFS) et la survie globale (OS) à 1 an était respectivement de $30 \%$ et $52 \%$ et l'OS médiane était de 12.9 mois ce qui est plus de deux fois supérieur aux résultats obtenus historiquement avec les chimiothérapies de 1re ligne [14]. Néanmoins, cette comparaison est basée sur des cohortes historiques rétrospectives et il n'existe aucun essai prospectif randomisé comparant l'efficacité de la chimiothérapie et de l'immunothérapie. Le traitement par avelumab était bien toléré puisque $5 \%$ d'effets indésirables de grade 3 ont été rapportés et aucun effet indésirable de grade 4 ou décès lié au traitement n'ont été observés. Les données d'efficacité à 2 ans présentées à l'ASCO 2018 (American Society of Clinical Oncology) rapportent un bénéfice durable avec une PFS et une OS à 2 ans de $26 \%$ et $36 \%$ respectivement. La démonstration de l'efficacité de l'avelumab en $2^{\mathrm{e}}$ ligne ou plus chez les patients avec carcinome à cellules de Merkel métastatique a conduit à son évaluation en $1^{\text {re }}$ ligne. Dans une étude de phase II incluant 39 patients avec carcinome à cellules de Merkel avancé traité par avelumab en première ligne, une analyse intermédiaire portant sur 29 patients avec au moins 3 mois de suivi a montré une efficacité majeure avec un ORR de $62 \%$ (RC : $14 \%$ et RP : $48 \%$ ). Devant ces résultats, l'avelumab a reçu l'approbation de la FDA (Food and Drug Administration) et de l'EMA (European Medical Association) en 2017 pour 
le traitement du carcinome à cellules de Merkel indépendamment de l'administration préalable ou non de chimiothérapie.

\section{Inhibiteurs de $P D-1$}

\section{Pembrolizumab}

Une étude de phase II incluant 26 patients avec carcinome à cellules de Merkel avancé (stade IIIb ou IV) traités par pembrolizumab $2 \mathrm{mg} / \mathrm{kg}$ toutes les 3 semaines en $1^{\text {re }}$ ligne a montré un ORR de $56 \%$ dont $16 \%$ de RC et $40 \%$ de RP [20]. Ces réponses étaient rapides puisque la durée médiane de réponse était de 2,8 mois (extrêmes : 1,5-9,7). Les données présentées à l'ASCO 2018 ont rapporté une PFS médiane de 16,8 mois et une OS médiane non atteinte, pour une médiane de suivi de 14,9 mois. À noter que la réponse au traitement n'était pas corrélée à l'expression de PD-1, ni au statut MCpyV. La tolérance était satisfaisante avec seulement $15 \%$ d'effet indésirable grade 3 ou 4.

\section{Nivolumab}

Une étude est en cours (ClinicalTrials.gov : NCT02488759) afin d'évaluer la tolérance et l'efficacité du nivolumab seul ou en association avec l'ipilimumab (1 mg/kg), un anti-LAG3 ou le daratumumab (anticorps monoclonal anti-CD38) chez les patients présentant un cancer viro-induits incluant des patients avec carcinome à cellules de Merkel métastatique. Une autre étude de phase II comparant l'efficacité du nivolumab en association à l'ipilimumab +/radiothérapie stéréotaxique dans les carcinomes à cellules de Merkel métastatiques est ouverte (NCT03071406). Plusieurs molécules en association avec le nivolumab sont actuellement à l'étude : le talimogene laherparepvec (NCT02978625), la combinaison d'un agoniste de TLR 7/8 (toll-like récepteur) intra-lésionnel et d'un agoniste de CD122 (NCT03435640) systémique ou encore un anticorps agoniste anti-OX40 (NCT03071757).

Par ailleurs, l'efficacité du nivolumab en indication néoadjuvante dans différents cancers viroinduits dont le carcinome à cellules de Merkel résécable est en cours (NCT02488759). Les résultats préliminaires présentés à l'ASCO 2018 rapportent une réduction tumorale radiologique et anatomopathologique de respectivement $40 \%$ et $65 \%$ après traitement néoadjuvant par nivolumab 4 semaines avant la chirurgie chez 25 patients présentant un carcinome à cellules de Merkel résécable de stade IIA-IV. Sur les 21 patients suivis après chirurgie, 19 ne présentaient pas de rechute tumorale à 1 an. D'autres investigations sont nécessaires afin de valider l'utilisation de l'immunothérapie par anti-PD1 en néoadjuvant.

\section{Thérapies conventionnelles}

\section{Chimiothérapie}

La chimiothérapie pour carcinome à cellules de Merkel métastatiques couramment utilisée est représentée par une association de sels de platine (carboplatine ou cisplatine) et d'etoposide, par le topotecan ou par une combinaison de cyclophosphamide, doxorubicine et vincristine. L'efficacité de ces différentes lignes de chimiothérapie a été évaluée dans des études rétrospectives mais aucun essai clinique prospectif randomisé n'a été conduit. Une revue récente de la littérature a rapporté des ORR élevés allant de $52 \%$ à $61 \%$ en $1^{\text {re }}$ ligne et $23 \%$ en deuxième ligne ou plus chez les patients présentant un carcinome à cellules de Merkel de stade IV traités par chimiothérapie $[14,15]$. Cependant, les réponses étaient de courte durée avec une médiane de réponse de seulement 6 mois indépendamment de la ligne de traitement, une PFS médiane de 3.1 mois en $1^{\text {re }}$ ligne versus 2 mois en $2^{\mathrm{e}}$ ligne et une OS médiane de 9 mois environ [14]. De plus, la toxicité de la chimiothérapie était élevée en particulier dans cette population cible d'âge $>65$ ans. Les effets indésirables les plus fréquents étaient la myélosuppression, le sepsis, l'asthénie, l'alopécie, les nausées et vomissements et 
l'insuffisance rénale. Le taux de mortalité liée à la chimiothérapie était de $7.7 \%$ dans une étude et parmi ces 9 décès, 6 étaient liés à la doxorubicine [33].

\section{$\underline{\text { Radiothérapie }}$}

Une littérature croissante suggère la possibilité d'un effet abscopal chez les patients traités par immunothérapie en lien avec une augmentation de la présentation d'antigènes et le recrutement de lymphocytes T CD8+ après irradiation. Une étude rétrospective récente a rapporté deux cas de réponse avec effet abscopal chez des patients ayant reçu une fraction de radiothérapie stéréotaxique complémentaire après progression sous anti-PD-1 pour carcinome à cellules de Merkel métastatique. Cependant, il n'existe actuellement pas de données claires sur la place de la radiothérapie en association avec l'immunothérapie dans le traitement du carcinome à cellules de Merkel.

\section{Autres stratégies thérapeutiques}

Bien que l'efficacité de l'immunothérapie soit majeure, $50 \%$ environ des patients ne répondent pas au blocage de l'axe anti-PD1/anti-PD-L-1 d'où la nécessité de développer de nouvelles thérapeutiques. Actuellement, de nouvelles approches immunologiques et nonimmunologiques sont en cours de développement.

\section{Immunothérapie}

\section{Ipilimumab}

Une étude rétrospective de 5 patients avec carcinome à cellules de Merkel métastatique traité par ipilimumab, un anticorps anti-CTLA4, a rapporté une efficacité modérée avec 2 cas de RC, 2 cas de stabilité et un cas de progression. D'autres études sont en cours afin d'évaluer l'efficacité de l'ipilimumab dans cette indication (NCT02488759, NCT03071406).

Néanmoins, la toxicité élevée de l'ipilimumab dans cette population cible âgée doit être prise en compte.

\section{Immunothérapie intra-lésionnelle}

Plusieurs cas de patients un carcinome à cellules de Merkel localement avancé ou métastatique traités par injection intra-lésionnelle de T-vec (talimogene laherparepvec) [34], TNF (tumor necrosis factor) [35,36] ou interferon [37,38] ont été publiés. Une étude évaluant l'efficacité du TTI-621 intra-tumoral seul ou en association à d'autres traitements est en cours (NCT02890368). Le T-vec intra-lésionnel seul ou en association à la radiothérapie (NCT02819843) ou au nivolumab (NCT02978625) est lui aussi en cours d'évaluation. La combinaison d'un anti-CTLA4, un anti-PDèL-1 et un agoniste de TLR3 intra-lésionnel est elle aussi à l'étude (NCT02643303).

\section{Autres immunothérapies systémiques}

Plusieurs nouvelles molécules sont en cours d'évaluation pour la prise en charge du carcinome à cellules de Merkel métastatique : un anticorps agoniste anti-OX40 (NCT03241173), un anticorps agoniste anti-GITR (Glucocorticoid-induced TNFR-related protein) (NCT03126110) et le F16IL2, une protéine de fusion recombinante constituée de la cytokine IL2 et d'un fragment d'anticorps humain spécifique de la tenascin-C, en association avec le paclitaxel (NCT02054884).

\section{Thérapie cellulaire}

Un patient présentant un carcinome à cellules de Merkel métastatique traité avec succès par perfusion de LT CD8+ ciblant le MCPyV a été décrit avec une réponse durable (535 jours) [39]. Une étude évaluant le traitement par LT CD8+ autologues dirigés contre le MCPyV en association à l'avelumab (NCT02584829) est en cours. 
De plus, l'efficacité de la thérapie cellulaire NK est en cours d'évaluation en association à l'IL15 chez les patients de stade IIIb et IV (NCT02465957) et en association métronomique avec une combinaison thérapeutique multiple chez les patients ayant progressé sous anti-PDL1 (NCT03167164).

\section{Les thérapies ciblées}

L'identification d'altérations génomiques au sein des cellules tumorales est une piste scientifique en cours de développement pour le traitement du carcinome à cellules de Merkel. Ainsi, dans une étude récente, les altérations moléculaires présentent chez 17 patients présentant un carcinome à cellules de Merkel ont été identifiées par NGS (next-generation sequencing) permettant d'identifier 60 altérations moléculaires distinctes ; les altérations les plus fréquentes impliquaient le gène TP53 et le cycle cellulaire (CDKN2A/B, CDKN2C et RB1) ainsi que la voie PI3K/AKT/mTor [40]. D'autres études précliniques sont donc nécessaires pour identifier les voies de signalisation impliquées dans le développement du carcinome à cellules de Merkel et guider la prescription d'éventuelles thérapies ciblées.

Une série de 5 patients présentant un carcinome à cellules de Merkel métastatique a rapporté une efficacité du traitement par inhibiteur de tyrosine kinase ciblant le VEGF (vascular endothelial growth factor) (pazopanib $n=4$ et cabozantinib $n=1$ ) [41]. Un cas de réponse après analogue de la somatostatine seul [42] et un cas en association avec un inhibiteur de mTor [43] ont été décrits. Plusieurs études sont en cours afin de tester l'efficacité de ces traitements (NCT02514824).

\section{Cas particulier des métastases cérébrales}

Les métastases cérébrales des carcinomes à cellules de Merkel sont extrêmement rares et peu de cas ont été rapportés dans la littérature. Une étude rétrospective récente a étudié les facteurs prédictifs de survie des patients présentant un carcinome à cellules de Merkel avec atteinte métastatique cérébrale à partir d'une série de 13 cas institutionnels et d'une revue de la littérature [48]. Sur les 40 patients inclus, 82,5\% avaient reçu un traitement par radiothérapie cérébrale, 59,5\% un traitement systémique et $35 \%$ une résection chirurgicale [48]. En analyse univariée, le traitement chirurgical, la radiothérapie cérébrale et l'atteinte métastatique cérébrale unique étaient tous associés à un bénéfice sur la survie globale [48]. Cependant, en analyse multivariée, seule la résection chirurgicale était un facteur prédictif indépendant de survie globale ( $\mathrm{HR}=0,12 ; \mathrm{p}=0,003)$ après ajustement sur l'âge, le volume tumoral et la radiothérapie cérébrale [48]. D'autres études prospectives sont donc nécessaires afin de définir la prise en charge optimale des patients avec localisations cérébrales de carcinome à cellules de Merkel.

Il n'existe actuellement aucune conférence de consensus concernant la prise en charge des métastases cérébrales de carcinome à cellules de Merkel. Tout patient présentant un carcinome à cellules de Merkel avec atteinte cérébrale métastatique doit donc faire l'objet d'une réunion de concertation pluridisciplinaire afin de définir un projet thérapeutique personnalisé prenant en compte les différentes possibilités thérapeutiques : chirurgie en cas d'atteinte mono- ou pauci-lésionnelle, radiothérapie cérébrale stéréotaxique, immunothérapie et chimiothérapie.

\section{Stratégie thérapeutique recommandée dans la prise en charge des carcinomes de Merkel métastatiques}

En $1^{\text {re }}$ ligne 
L'inclusion dans un protocole de recherche clinique doit être systématiquement recherchée au diagnostic de carcinome à cellules de Merkel métastatique et représente l'option thérapeutique à privilégier lorsque le patient est éligible [22]. Dans le cas contraire, un traitement systémique par immunothérapie anti-PD-1 ou anti-PD-L-1 éventuellement associé à une radiothérapie ou chirurgie complémentaire doit être discuté au sein d'une réunion de concertation pluridisciplinaire [22]. Depuis septembre 2017, l'avelumab bénéficie d'une AMM (autorisation de mise sur le marché) en Europe dans le traitement du carcinome à cellules de Merkel métastatique indépendamment de la ligne de traitement mais son remboursement dans cette indication est en attente. Les anti-PD1, pembrolizumab et nivolumab, n'ont actuellement pas encore obtenu l'AMM en Europe. Aux États-Unis, bien que seul l'avelumab ait été approuvé par la FDA dans le traitement du carcinome à cellules de Merkel métastatique, les recommandations du NCCN (Clinical Practice Guidelines in Oncology) pour le traitement du carcinome à cellules de Merkel métastatique en $1^{\text {re }}$ ligne précisent que le choix de l'avelumab, du nivolumab ou du pembrolizumab est laissé à l'appréciation du clinicien.

Les résultats impressionnants des études évaluant le blocage de l'axe antiPD-1/PD-L-1 ont changé le pronostic et la prise en charge des patients présentant un carcinome à cellules de Merkel métastatique. L'efficacité de l'immunothérapie est majeure avec des réponses rapides et durables $[16,17,19,32]$. Les résultats des bénéfices à long terme sont attendus et une amélioration significative de la survie, est espérée. Bien qu'aucune étude randomisée prospective n'ait démontré la supériorité de l'immunothérapie sur la chimiothérapie dans le traitement des carcinomes à cellules de Merkel métastatiques, les taux de réponses observés avec l'immunothérapie étaient similaires à ceux obtenus avec la chimiothérapie mais ces réponses étaient plus durables. De plus, le profil de tolérance était excellent et largement supérieur à celui de la chimiothérapie [16-18]. Enfin, l'efficacité de l'immunothérapie était supérieure en $1^{\text {re }}$ ligne comparée aux réponses après progression sous chimiothérapie [16-18]. Ces arguments contribuent définir les anti-PD-L-1 et anti-PD-1 comme la stratégie thérapeutique de $1^{\text {re }}$ ligne pour les patients avec carcinome à cellules de Merkel avancé [22] en l'absence de contre-indications (immunosuppression, transplantation d'organes, pathologies auto-immunes). Il n'existe actuellement aucun argument pour préférer une molécule à une autre puisque l'efficacité et la tolérance semblent globalement similaires $[16,20]$ bien qu' aucune étude comparative n'ait été réalisée. Cependant, l'avelumab a été plus largement exploré et est le seul à avoir obtenu une approbation dans cette indication. De plus, un bénéfice en termes de qualité de vie a été démontré avec l'avelumab uniquement [19]. Toutefois, le pembrolizumab et le nivolumab restent deux stratégies thérapeutiques de $1^{\text {re }}$ ligne largement acceptables [44]. Bien que l'efficacité d'un switch des anti-PD-1 vers les antiPD-L-1 en cas de progression n'ait pas été exploré, cette stratégie ne semble pas recommandée au vu des données obtenues dans le traitement du mélanome [45].

L'utilisation de la chimiothérapie en $1^{\text {re }}$ ligne n'est pas recommandée à moins que le patient ne présente une contre-indication à l'immunothérapie ou n'ait progressé sous immunothérapie [22].

Bien que l'efficacité de l'immunothérapie soit majeure, $50 \%$ environ des patients ne répond pas au blocage de l'axe anti-PD1/anti-PD-L-1 d'où la nécessité d'identifier des facteurs prédictifs de réponse. La réponse à l'immunothérapie par anti-PD-L-1 et anti-PD-1 ne semble pas corrélée au statut MCPyV de la tumeur, ni à l'expression de PD-L1 par le microenvironnement tumoral [17,20]. L'intérêt de tester le statut MCPyV (par immunohistochimie ou sérologie) ainsi que l'évaluation du taux d'expression de PD-L-1 (par 
immunohistochimie) apparaît donc limité en routine. D'autres études sont nécessaires afin d'identifier de nouveaux biomarqueurs.

\section{Après échec du blocage de l'axe PD-1/PD-L-1}

Pour les patients ayant progressé sous anti-PD-1 ou anti-PD-L-1 ou présentant une contreindication à l'immunothérapie, le NCCN recommande l'usage d'une chimiothérapie conventionnelle après validation pluridisciplinaire [22]. Les options envisageables sont : cisplatine +/- etoposide, carboplatine +/- etoposide, topotecan ou l'association cyclophosphamide, doxorubicine (ou epirubicine) et vincristine [14,22]. Il n'existe pas d'études démontrant la supériorité d'un protocole de chimiothérapie par rapport à un autre, ni de recommandations précises sur la séquence optimale de traitement à privilégier.

De nombreuses études cliniques évaluant de nouvelles molécules thérapeutiques sont en cours et les résultats pourront très probablement élargir les options thérapeutiques à la disposition du clinicien dans le traitement du carcinome à cellules de Merkel métastatique.

\section{Centre expert}

Toute interrogation concernant la prise en charge diagnostique ou thérapeutique d'un patient présentant un probable carcinome à cellules de Merkel peut être adressée au centre expert régional du réseau CARADERM. Créé à l'initiative de l'INCa (Institut National du Cancer), ce réseau constitué de services d'onco-dermatologie et anatomopathologie expert en tumeurs rares cutanées fournit une relecture anatomopathologique en cas de doute diagnostique et organise des réunions de concertation pluridisciplinaire pour la discussion des cas les plus complexes.

\section{Surveillance}

Le risque de récurrence des carcinomes à cellules de Merkel est élevé puisque la survie sans récidive à 5 ans des patients présentant un carcinome à cellules de Merkel varie de 25 à $50 \%$ environ selon les études $[10,11,46]$. Le NCCN recommande un examen clinique cutané et ganglionnaire tous les 3 à 6 mois pendant les 3 premières années puis tous les 6 mois à 1 an au long cours [22]. Bien qu'une surveillance radiologique par IRM cérébrale et scanner thoraco-abdomino-pelvien ou TEP au18FDG soit généralement considérée comme appropriée en pratique clinique pour les patients à haut risque de récidive, il n'existe aucune conférence de consensus permettant d'identifier une population devant bénéficier d'une surveillance radiologique, principalement en raison du manque de données prospectives sur la survie sans récurrence des patients présentant un carcinome à cellules de Merkel. Le titrage des anticorps anti-T dirigés contre les oncoprotéines virales du MCPyV au diagnostic puis tout au long du suivi peut aider à la surveillance mais n'est pas disponible en routine. En effet, les patients séropositifs au diagnostic présentaient un risque de récurrence inférieur et après traitement, les patients séropositifs répondeurs présentaient une diminution rapide du titre d'anticorps. En cas de récurrence, il était observé une ré-ascension précoce de ce taux [47].

\section{Conclusion}

Le carcinome à cellules de Merkel est un cancer agressif dont le pronostic et la prise en charge thérapeutique ont été récemment bouleversés par l'arrivée de l'immunothérapie. Le blocage de l'axe anti-PD-1 / anti-PD-L-1 représente actuellement le traitement de première 
intention des carcinomes à cellules de Merkel métastatiques avec des réponses prolongées et une tolérance satisfaisante. Cependant, environ $50 \%$ des patients ne répondent pas à ce traitement et il existe un besoin urgent d'identifier des facteurs prédictifs de réponse à l'immunothérapie. Les options thérapeutiques après échec de l'immunothérapie sont limitées à la chimiothérapie avec un bénéfice faible sur la survie et de nombreuses molécules sont actuellement étudiées afin de fournir d'autres options thérapeutiques efficaces.

\section{Bibliographie}

[1] Reichgelt BA, Visser O. Epidemiology and survival of Merkel cell carcinoma in the Netherlands. A population-based study of 808 cases in 1993-2007. Eur J Cancer 2011;47:579-85.

[2] Albores-Saavedra J, Batich K, Chable-Montero F, Sagy N, Schwartz AM, Henson DE. Merkel cell carcinoma demographics, morphology, and survival based on 3870 cases: a population based study. J Cutan Pathol 2010;37:20-7.

[3] Zaar O, Gillstedt M, Lindelöf B, Wennberg-Larkö A-M, Paoli J. Merkel cell carcinoma incidence is increasing in Sweden. J Eur Acad Dermatol Venereol 2016;30:1708-13.

[4] Paulson KG, Park SY, Vandeven NA, Lachance K, Thomas H, Chapuis AG, et al. Merkel cell carcinoma: Current US incidence and projected increases based on changing demographics. J Am Acad Dermatol 2018;78:457-463.e2.

[5] Stang A, Becker JC, Nghiem P, Ferlay J. The association between geographic location and incidence of Merkel cell carcinoma in comparison to melanoma: An international assessment. Eur J Cancer 2018;94:47-60.

[6] Feng H, Shuda M, Chang Y, Moore PS. Clonal integration of a polyomavirus in human Merkel cell carcinoma. Science 2008;319:1096-100.

[7] Becker JC, Stang A, DeCaprio JA, Cerroni L, Lebbé C, Veness M, et al. Merkel cell carcinoma. Nat Rev Dis Primers 2017;3:17077.

[8] Moshiri AS, Doumani R, Yelistratova L, Blom A, Lachance K, Shinohara MM, et al. Polyomavirus-Negative Merkel Cell Carcinoma: A More Aggressive Subtype Based on Analysis of 282 Cases Using Multimodal Tumor Virus Detection. J Invest Dermatol 2017;137:819-27.

[9] Lebbe C, Becker JC, Grob J-J, Malvehy J, Del Marmol V, Pehamberger H, et al. Diagnosis and treatment of Merkel Cell Carcinoma. European consensus-based interdisciplinary guideline. Eur J Cancer 2015;51:2396-403.

[10] Fields RC, Busam KJ, Chou JF, Panageas KS, Pulitzer MP, Allen PJ, et al. Five hundred patients with Merkel cell carcinoma evaluated at a single institution. Ann Surg 2011;254:465-473; discussion 473-475.

[11] Allen PJ, Bowne WB, Jaques DP, Brennan MF, Busam K, Coit DG. Merkel cell carcinoma: prognosis and treatment of patients from a single institution. J Clin Oncol 2005;23:2300-9. 
[12] Harms KL, Healy MA, Nghiem P, Sober AJ, Johnson TM, Bichakjian CK, et al. Analysis of Prognostic Factors from 9387 Merkel Cell Carcinoma Cases Forms the Basis for the New 8th Edition AJCC Staging System. Ann Surg Oncol 2016;23:356471.

[13] Kouzmina M, Koljonen V, Leikola J, Böhling T, Lantto E. Frequency and locations of systemic metastases in Merkel cell carcinoma by imaging. Acta Radiol Open 2017;6:2058460117700449.

[14] Nghiem P, Kaufman HL, Bharmal M, Mahnke L, Phatak H, Becker JC. Systematic literature review of efficacy, safety and tolerability outcomes of chemotherapy regimens in patients with metastatic Merkel cell carcinoma. Future Oncol 2017;13:1263-79.

[15] Iyer JG, Blom A, Doumani R, Lewis C, Tarabadkar ES, Anderson A, et al. Response rates and durability of chemotherapy among 62 patients with metastatic Merkel cell carcinoma. Cancer Med 2016;5:2294-301.

[16] D’Angelo SP, Russell J, Lebbé C, Chmielowski B, Gambichler T, Grob J-J, et al. Efficacy and Safety of First-line Avelumab Treatment in Patients With Stage IV Metastatic Merkel Cell Carcinoma: A Preplanned Interim Analysis of a Clinical Trial. JAMA Oncol 2018;4(9):e180077.

[17] Kaufman HL, Russell J, Hamid O, Bhatia S, Terheyden P, D’Angelo SP, et al. Avelumab in patients with chemotherapy-refractory metastatic Merkel cell carcinoma: a multicentre, single-group, open-label, phase 2 trial. Lancet Oncol 2016;17:1374-85.

[18] Kaufman HL, Russell JS, Hamid O, Bhatia S, Terheyden P, D’ Angelo SP, et al. Updated efficacy of avelumab in patients with previously treated metastatic Merkel cell carcinoma after $\geq 1$ year of follow-up: JAVELIN Merkel 200, a phase 2 clinical trial. J Immunother Cancer 2018;6(1):7.

[19] Kaufman HL, Hunger M, Hennessy M, Schlichting M, Bharmal M. Nonprogression with avelumab treatment associated with gains in quality of life in metastatic Merkel cell carcinoma. Future Oncol 2018;14:255-66

[20] Nghiem PT, Bhatia S, Lipson EJ, Kudchadkar RR, Miller NJ, Annamalai L, et al. PD-1 Blockade with Pembrolizumab in Advanced Merkel-Cell Carcinoma. N Engl J Med 2016;374:2542-52.

[21] Ellis DL, Davis RS. Evidence-based management of primary and localized Merkel cell carcinoma: a review. Int J Dermatol 2013;52:1248-58.

[22] Bichakjian CK, Olencki T, Aasi SZ, Alam M, Andersen JS, Blitzblau R, et al. Merkel Cell Carcinoma, Version 1.2018, NCCN Clinical Practice Guidelines in Oncology. J Natl Compr Canc Netw 2018;16:742-74.

[23] Veness M, Howle J. Radiotherapy alone in patients with Merkel cell carcinoma: the Westmead Hospital experience of 41 patients. Australas J Dermatol 2015;56:19-24.

[24] Gunaratne DA, Howle JR, Veness MJ. Sentinel lymph node biopsy in Merkel cell carcinoma: a 15-year institutional experience and statistical analysis of 721 reported cases. Br J Dermatol 2016;174:273-81.

[25] Stokes JB, Graw KS, Dengel LT, Swenson BR, Bauer TW, Slingluff CL, et al. Patients with Merkel cell carcinoma tumors < or $=1.0 \mathrm{~cm}$ in diameter are unlikely to harbor regional lymph node metastasis. J Clin Oncol 2009;27:3772-7. 
[26] Kachare SD, Wong JH, Vohra NA, Zervos EE, Fitzgerald TL. Sentinel lymph node biopsy is associated with improved survival in Merkel cell carcinoma. Ann Surg Oncol 2014;21:1624-30.

[27] Servy A, Maubec E, Sugier PE, Grange F, Mansard S, Lesimple T, et al. Merkel cell carcinoma: value of sentinel lymph-node status and adjuvant radiation therapy. Ann Oncol 2016;27:914-9.

[28] Hasan S, Liu L, Triplet J, Li Z, Mansur D. The role of postoperative radiation and chemoradiation in merkel cell carcinoma: a systematic review of the literature. Front Oncol 2013;3:276.

[29] Bhatia S, Storer BE, Iyer JG, Moshiri A, Parvathaneni U, Byrd D, et al. Adjuvant Radiation Therapy and Chemotherapy in Merkel Cell Carcinoma: Survival Analyses of 6908 Cases From the National Cancer Data Base. J Natl Cancer Inst 2016;108.

[30] Jouary T, Leyral C, Dreno B, Doussau A, Sassolas B, Beylot-Barry M, et al. Adjuvant prophylactic regional radiotherapy versus observation in stage I Merkel cell carcinoma: a multicentric prospective randomized study. Ann Oncol 2012;23:1074-80.

[31] Veness MJ, Perera L, McCourt J, Shannon J, Hughes TM, Morgan GJ, et al. Merkel cell carcinoma: improved outcome with adjuvant radiotherapy. ANZ J Surg 2005;75:275-81.

[32] Shirley M. Avelumab: A Review in Metastatic Merkel Cell Carcinoma. Target Oncol 2018;13:409-16.

[33] Voog E, Biron P, Martin JP, Blay JY. Chemotherapy for patients with locally advanced or metastatic Merkel cell carcinoma. Cancer 1999;85:2589-95.

[34] Blackmon JT, Dhawan R, Viator TM, Terry NL, Conry RM. Talimogene laherparepvec for regionally advanced Merkel cell carcinoma: A report of 2 cases. JAAD Case Rep 2017;3:185-9.

[35] Ito Y, Kawamura K, Miura T, Ueda K, Onodera H, Takahashi H, et al. Merkel cell carcinoma. A successful treatment with tumor necrosis factor. Arch Dermatol 1989;125:1093-5.

[36] Hata Y, Matsuka K, Ito O, Matsuda H, Furuichi H, Konstantinos A, et al. Two cases of Merkel cell carcinoma cured by intratumor injection of natural human tumor necrosis factor. Plast Reconstr Surg 1997;99:547-53.

[37] Biver-Dalle C, Nguyen T, Touzé A, Saccomani C, Penz S, Cunat-Peultier S, et al. Use of interferon-alpha in two patients with Merkel cell carcinoma positive for Merkel cell polyomavirus. Acta Oncol 2011;50:479-80.

[38] Wahl RU, Braunschweig T, Ghassemi A, Rübben A. Immunotherapy with imiquimod and interferon alfa for metastasized Merkel cell carcinoma. Curr Oncol 2016;23:e150153.

[39] Chapuis AG, Afanasiev OK, Iyer JG, Paulson KG, Parvathaneni U, Hwang JH, et al. Regression of metastatic Merkel cell carcinoma following transfer of polyomavirusspecific T cells and therapies capable of re-inducing HLA class-I. Cancer Immunol Res 2014;2:27-36. 
[40] Cohen PR, Tomson BN, Elkin SK, Marchlik E, Carter JL, Kurzrock R. Genomic portfolio of Merkel cell carcinoma as determined by comprehensive genomic profiling: implications for targeted therapeutics. Oncotarget 2016;7:23454-67.

[41] Tarabadkar ES, Thomas H, Blom A, Parvathaneni U, Olencki T, Nghiem P, et al. Clinical Benefit from Tyrosine Kinase Inhibitors in Metastatic Merkel Cell Carcinoma: A Case Series of 5 Patients. Am J Case Rep 2018;19:505-11.

[42] Fakiha M, Letertre P, Vuillez JP, Lebeau J. Remission of Merkel cell tumor after somatostatin analog treatment. J Cancer Res Ther 2010;6:382-4.

[43] Slovacek L. Merckel cell carcinoma of the skin treated with somatostatin analogue and mTOR inhibitor exhausted after primary surgery, adjuvant radiotherapy and palliative chemotherapy. Bratisl Lek Listy 2014;115:663-5.

[44] Paulson KG, Bhatia S. Advances in Immunotherapy for Metastatic Merkel Cell Carcinoma: A Clinician’s Guide. J Natl Compr Canc Netw 2018;16:782-90.

[45] Kim JM, Chen DS. Immune escape to PD-L1/PD-1 blockade: seven steps to success (or failure). Ann Oncol 2016;27:1492-504.

[46] Fields RC, Busam KJ, Chou JF, Panageas KS, Pulitzer MP, Kraus DH, et al. Recurrence and survival in patients undergoing sentinel lymph node biopsy for merkel cell carcinoma: analysis of 153 patients from a single institution. Ann Surg Oncol 2011;18:2529-37.

[47] Paulson KG, Lewis CW, Redman MW, Simonson WT, Lisberg A, Ritter D, et al. Viral oncoprotein antibodies as a marker for recurrence of Merkel cell carcinoma: A prospective validation study. Cancer 2017;123:1464-74.

[48] Harary M, Kavouridis VK, Thakuria M, Smith TR. Predictors of survival in neurometastatic Merkel cell carcinoma. Eur J Cancer 2018;101:152-9. 
Table 1 : Etudes évaluant l'efficacité et la tolérance des anti-PD1 et anti-PD-L1 dans le carcinome de Merkel métastatique

\begin{tabular}{|c|c|c|c|c|}
\hline & $\begin{array}{c}\text { Avelumab } \\
\text { (Kaufman et al.) } \\
{[17,18]} \\
\text { JAVELIN MERKEL } \\
200 \text { PART A } \\
\text { ASCO } 2018\end{array}$ & $\begin{array}{c}\text { Avelumab } \\
\text { (D’Angelo et al.) } \\
\text { [16] } \\
\text { JAVELIN MERKEL } \\
200 \text { PART B }\end{array}$ & $\begin{array}{c}\text { Pembrolizumab } \\
\text { (Nghiem et al.) } \\
\text { [20] } \\
\text { ASCO } 2018\end{array}$ & $\begin{array}{c}\text { Nivolumab } \\
\text { (Topalian et al.) } \\
\text { Abstract CT074 } \\
\text { AACR } 2017\end{array}$ \\
\hline Etude & Phase II & Phase II & Phase II & Phase I - II \\
\hline Population & Stade IV & Stade IV & Stade IIIb ou IV & Stade IV \\
\hline $\begin{array}{l}\text { Lignes de } \\
\text { traitement }\end{array}$ & $\begin{array}{c}\geq 2 \text { 2ème ligne } \\
\text { Echec d'au moins } \\
\text { une ligne de } \\
\text { chimiothérapie }\end{array}$ & $1^{\text {ère }}$ ligne & $1^{\text {ère }}$ ligne & $\begin{array}{c}60 \% \text { en } 1^{\text {ère }} \text { ligne } \\
\text { Maximum } 2 \\
\text { lignes } \\
\text { précédentes }\end{array}$ \\
\hline $\mathrm{N}$ patients & 88 patients & 39 patients & 50 patients & 25 patients \\
\hline $\begin{array}{l}\text { Médiane de suivi } \\
\text { (extrêmes) }\end{array}$ & $\begin{array}{c}29,2 \text { mois } \\
(24,8-38,1)\end{array}$ & $\begin{array}{c}5,1 \text { mois } \\
(0,3-11,3)\end{array}$ & $\begin{array}{c}14,9 \text { mois } \\
(0,4-36)\end{array}$ & 51 semaines \\
\hline $\begin{array}{l}\text { ORR (\%) } \\
\text { Dont CR (\%) }\end{array}$ & $\begin{array}{c}33 \% \\
\text { (Dont 11\%) } \\
\end{array}$ & $\begin{array}{c}62 \% \\
\text { (Dont 14\%) }\end{array}$ & $\begin{array}{c}56 \% \\
\text { (Dont 24\%) }\end{array}$ & $\begin{array}{c}68 \% \\
\text { (Dont 14\%) }\end{array}$ \\
\hline PFS & $\begin{array}{c}\text { Médiane: } \\
2,7 \text { mois } \\
\text { A } 1 \text { an : } 30 \% \\
\text { A } 2 \text { ans : } 26 \% \\
\end{array}$ & $\begin{array}{l}\text { Médiane: } \\
9,1 \text { mois }\end{array}$ & $\begin{array}{c}\text { Médiane: } \\
16,8 \text { mois } \\
\text { A } 1 \text { an : } 52 \% \\
\text { A } 2 \text { ans : } 48 \% \\
\end{array}$ & $\begin{array}{l}\text { Médiane : NA } \\
\text { A } 6 \text { mois : } 75 \%\end{array}$ \\
\hline OS & $\begin{array}{c}\text { Médiane : } \\
12,9 \text { mois } \\
\text { A } 1 \text { an : } 52 \% \\
\text { A } 2 \text { ans : } 36 \%\end{array}$ & - & $\begin{array}{l}\text { Médiane : NA } \\
\text { A } 1 \text { an : } 72 \% \\
\text { A } 2 \text { ans : } 69 \%\end{array}$ & $\begin{array}{l}\text { Médiane : NA } \\
\text { A } 6 \text { mois : } 80 \%\end{array}$ \\
\hline El G3-4 & $11 \%$ & $20 \%$ & $28 \%$ & $20 \%$ \\
\hline
\end{tabular}

$\mathbf{N}$ : nombre ; ORR : taux de réponse objective ; PFS : survie sans progression ; OS : survie globale ; EI G3-4 : effet indésirable grade 3-4 ; NA : non atteinte ; ASCO : American Society of Clinical Oncology ; AACR : American Association for Cancer Research. 